\title{
María Victoria Peralta. Su compromiso con la educación parvularia en Chile y Latinoamérica
}

Maria Victoria Peralta. Her commitment to kindergarten education in Chile and Latin America

Maria Victoria Peralta. Seu compromisso com a educação pré-escolar no Chile e na América Latina

Maria Victoria Peralta. Son engagement en faveur de l'enseignement maternel au Chili et en Amérique latine

\author{
Jaime Caiceo Escudero \\ Académico Universidad de Santiago de Chile \\ https://orcid.org/0000-0002-2808-140X \\ jcaiceo@ hotmail.com
}

\section{Resumen}

Chile posee la cobertura de todo su sistema escolar -básico y medio- pero no así de la educación parvularia, alcanzando solo el $50 \%$. El estado funda el primer kindergarten público solo en 1906. Su mayor desarrollo, sin embargo, ha sido en los últimos 50 años, gracias a la creación de dos organismos públicos: JUNJI e INTEGRA. En este último tiempo, María Victoria Peralta Espinosa ha jugado un importante rol en pro de la educación parvularia, transformándose en una figura señera en este nivel educacional, tanto en Chile como en Latinoamérica. Se pretende reconstituir su biografía y describir su acción educativa en pro de los derechos que la mujer madre tiene con sus hijos respecto a su enseñanza desde la más tierna infancia, a fin de lograr la cobertura total de este sector educacional.

Palabras clave: Educación Parvularia. Formación de educadoras de infantes. Políticas Públicas. Cobertura de la primera infancia. 


\begin{abstract}
Chile has coverage of its entire school system -basic and a half- but not so of kindergarten, reaching only $50 \%$. The state founded the first public kindergarten only in 1906. Its greatest development, however, has been in the last 50 years, thanks to the creation of two public bodies: JUNJI and INTEGRA. In the last time, Maria Victoria Peralta Espinosa has played an important role in the pursuit of kindergarten education, becoming an important figure at this educational level, both in Chile and Latin America. It is intended to reconstitute her biography and describe her educational action in the way of the rights that female mother has with her children with regard to her teaching from the earliest childhood, in order to achieve full coverage of this educational sector.
\end{abstract}

Keywords: Kindergarten. Infant education formation. Public Policy. Early childhood coverage.

\title{
Resumo
}

O Chile possui a cobertura de todo o seu sistema escolar básico e médio, mas não tanto na a educação pré-escolar, alcançando apenas os 50\%. O Estado funda o primeiro jardim de infância público só em 1906. Seu maior desenvolvimento, no entanto, ocorre nos últimos 50 anos, graças à criação de dois órgãos públicos: JUNJI e INTEGRA. Nesta última vez, Maria Victoria Peralta Espinosa desempenhou um papel importante a favor da educação pré-escolar, transformandose em uma figura de liderança nesse nível educacional, tanto no Chile quanto na América Latina. O objetivo é reconstituir sua biografia e descrever sua ação educacional em favor dos direitos que a mulher-mãe tem com seus filhos em relação ao ensino desde a primeira infância, a fim de obter uma cobertura completa desse setor educacional.

Palavras-chave: Educação Pré-escolar. Formação de educadores infantis. Políticas Públicas. Cobertura da primeira infância.

\section{Résumé}

Le Chili a la couverture de l'ensemble de son système scolaire -de base et moyen- mais pas de l'enseignement maternel, qui n'atteint que 50\%. L'État n'a fondé le premier jardin d'enfants public qu'en 1906. Son plus grand développement, cependant, a été au cours des 50 dernières années, grâce à la création de deux organismes publics : JUNJI et INTEGRA. Ces derniers temps, María Victoria Peralta Espinosa a joué un rôle important dans la promotion de l'enseignement maternel, devenant une figure de proue de ce niveau d'enseignement, tant au Chili qu'en Amérique latine. L'objectif est de reconstituer sa biographie et de décrire son action éducative en faveur des droits que les femmes mères ont avec leurs enfants en ce qui concerne leur éducation dès le plus jeune enfance, afin de parvenir à une couverture complète de ce secteur éducatif.

Mots-clés: L'enseignement maternel. La formation des éducatrices infantiles. Les Politiques Publiques. La couverture de la petite enfance. 


\section{Introducción}

En Chile existe formalmente la educación de la primera infancia desde 1864 (ARANCIBIA ET AL., 2004) y en 1906 se funda el primer kindergarten público (PERALTA, 2006). Su mayor desarrollo, sin embargo, ha sido en los últimos 50 años, gracias a la creación de dos organismos públicos: JUNJI ${ }^{1}$ e INTEGRA ${ }^{2}$. Durante su carrera profesional María Victoria Peralta Espinosa ha jugado un importante rol en pro de la educación parvularia. En el contexto anterior es que el objetivo de esta ponencia consiste en reconstruir su biografía y en dar a conocer el aporte que en este último tiempo, le ha hecho esta educadora a la mujer trabajadora al promover la educación universal de la primera infancia en Chile y su constante preocupación por la educación parvularia, tanto en su país como en Latinoamérica; de esta forma, se persigue comprender por qué fue galardonada en Chile con el Premio Nacional en Ciencias de la Educación 2019.

Este estudio se inscribe en el paradigma cualitativo en el contexto de la epistemología de las ciencias sociales y, en este caso, con la metodología propia de los estudios históricos, recurriendo a fuentes primarias y secundarias; entre ellas entrevistas a la propia educadora y a personas que han trabajado con ella; es decir, se trata de un abordaje biográfico con sus trayectorias (CORNEJO, 2006) y análisis de documentos. En este caso, se asume la división que Mario Bunge ${ }^{3}$ realiza sobre las ciencias sociales continuando la línea de Wilhelm Dilthey que realiza la gran clasificación entre ciencias de la naturaleza y ciencias del espíritu, privilegiando estas últimas como las que comprenden los fenómenos; como habrá descripción y análisis interpretativo de hechos y documentos, habrá una episteme cercana a la fenomenología y a la hermenéutica (CAICEO, 2018).

Es necesario tener presente que las instituciones públicas existentes para atender la educación parvularia en Chile no alcanzan en la actualidad a cubrir el 50\% de las necesidades del país; es el déficit que tiene la educación chilena, pues la cobertura del sistema escolar es casi total, existiendo el financiamiento público para todas las escuelas y liceos necesarios. Por lo mismo, la Dra. María Victoria Peralta ha estado promoviendo la sala cuna universal y la educación de la primera infancia en su totalidad para que el estado se haga responsable de una necesidad social y de desarrollo más pleno de niños y niñas. En efecto, socialmente la mujer en el mundo actual trabaja y necesita un lugar seguro y gratuito para dejar a sus hijos e hijas durante su día laboral; la psicología, por su parte, señala que el ser humano tiene su mayor capacidad de aprendizaje antes de los 7 años $^{4}$. La Dra. Peralta es la educadora de párvulos que más ha luchado en las últimas cuatro décadas, no solo en Chile sino que en los demás países del continente, por los derechos que la mujer madre tiene con sus hijos respecto a su educación desde la más tierna infancia. Además, se ha dedicado a reconstruir la historia de la educación parvularia en Chile y Latinoamérica. Por todo lo anterior, fue reconocida recientemente por el estado chileno al otorgarle el Premio Nacional de Ciencias de la Educación 2019 porque "ha realizado un importante trabajo de formación inicial y capacitación docente en el país y en

\footnotetext{
${ }^{1}$ Junta Nacional de Jardines Infantiles.

${ }^{2}$ Institución fundada en 1990 perteneciente a la Red de Fundaciones de la Presidencia de la República con la finalidad de integrar a los niños de la primera infancia más desfavorecidos a una labor lúdico-educativa.

${ }^{3}$ Famoso físico, filósofo y epistemólogo argentino muerto recientemente en Canadá a los 100 años: 24 de febrero de 2020 .

4 "Existe amplio consenso científico, por lo menos desde hace medio siglo, reafirmado más recientemente por las ciencias cognitivas y las neurociencias, en que los primeros años de vida constituyen un período crucial en el desarrollo futuro de las personas. El creciente posicionamiento de la infancia temprana en las políticas públicas y las agendas gubernamentales, ha traído una renovación y ampliación de ese consenso, y nuevas evidencias, las que se refieren a cómo el ambiente influye en las potencialidades del niño y la niña y, a cómo el bienestar en esta etapa temprana genera beneficios a largo plazo para las economías y las sociedades" (SUBSECRETARÍA DE EDUCACIÓN PARVULARIA, 2018, p. 9).
} 
Latinoamérica, asesorías en políticas públicas y programas para la primera infancia [...] y trabajos como consultora de $\mathrm{UNICEF}^{5}, \mathrm{UNESCO}^{6}, \mathrm{BID}^{7}, \mathrm{PMA}^{8}$ y $\mathrm{OEI}^{9,}$ (AA, 2019, s/p).

\section{1.- Principales Rasgos Biográficos de María Victoria Peralta Espinosa}

\section{a) Su infancia y su formación escolar}

María Victoria nació en Santiago de Chile el 26 de octubre 1949. Sus progenitores fueron doña Victoria Espinosa Lillo, egresada de Derecho de la Universidad de Chile y don Aníbal Peralta Fuentes, Coronel de Ejército, experto en Telecomunicaciones. Es hija única (PERALTA, 2020).

A los 2 años ingresó a un jardín infantil en jornada completa, pues su madre trabajaba; se ubicaba en calle Sazie de la capital. Luego ingresó al sistema escolar en el Cambridge College, en donde realizó la educación primaria y las humanidades hasta $5^{\circ}$ año, pues por razones de salud, debió aprobar el $6^{\circ}$ año de humanidades ${ }^{10}$ como alumna libre con exámenes en el Liceo $\mathrm{N}^{\circ} 1$ de Niñas, ubicado en el centro de la ciudad. De esa época recuerda a la profesora Guillermina de Biología, "por su atención a aspectos personales de sus estudiantes y además buena profesora de su ramo"; la misma opinión guarda de su profesora de Historia; también a las directoras del Colegio: "Luisa Vicentini, después supe que había sido dirigente feminista comunista" y a "Mireya Latorre Vicentini, su hija, quien era cálida en su dirección” (PERALTA, 2020, p. 1).

\section{b) Su formación universitaria}

Por su salud delicada y por consejo médico, al postular a la universidad, debió elegir "una carrera no muy extensa, que no me exigiera mucho. Analicé las que tenían esas características y elegí: Educación Parvularia, Bibliotecología y Técnico en Diseño textil" (PERALTA, 2020, p. 1). Sin embargo, su vocación por la primera infancia apareció rápidamente, convirtiéndose en la labor que más la ha desarrollado como persona, desde el punto de vista individual, y comprometida por los más pequeños durante toda su existencia profesional, en una perspectiva social: "Una vez que ingresé a Educación Parvularia, y escuché a mis maestras, no tuve ninguna duda, que era una de las carreras más importantes para el país y muy interesante por la formación integral que entregaba" (PERALTA, 2020, p. 1).

Postuló a la Universidad de Chile porque allí se daba la carrera de Educadora de Párvulos ${ }^{11}$, que era su primera opción, porque esa universidad "era más conocida [...] y, además que yo vivía en Santiago, y estaba cerca de mi casa, en Eleodoro Yáñez casi al llegar a José Miguel Infante" (PERALTA, 2020, p. 2). De esa época, recuerda a las académicas que más la marcaron:

Rebeca Soltanovich, Directora Inteligente, cálida, actualizada, estudiosa, comprometida, muy completa como persona. Linda Volosky, Profesora comprometida, dulce, alegre, responsable. Pilar Mira e Ingeborg de Banderas, profesoras de Música y Trabajos Manuales, buenas profesoras y

\footnotetext{
${ }^{5}$ Fondo de las Naciones Unidas para la Infancia.

${ }^{6}$ Organización de las Naciones Unidas para la Educación, la Ciencia y la Cultura.

${ }^{7}$ Banco Interamericano de Desarrollo.

${ }^{8}$ Programa Mundial de Alimentos.

${ }^{9}$ Organización de Estados Iberoamericanos.

${ }^{10}$ Ella estudió antes de que entrara en la vigencia la reforma de 1965, en que la educación primaria de 6 años se elevó a 8 con el nombre de educación básica y la educación secundaria de 6 años se disminuyó como educación media a 4.

${ }^{11}$ Carrera dependiente de la Facultad de Filosofía y Educación fundada el 22 de noviembre 1951 por Decreto Universitario $\mathrm{N}^{\circ}$ 1.238, promovida por la gran educadora Amanda Labarca, quien fue su primera Directora (CAICEO, 2015). La otra universidad que en esa época dictaba esta carrera era la Universidad de Concepción, ubicaba a 500 kilómetros al sur de la capital, lugar de residencia de la joven María Victoria.
} 
cálidas. Dina Alarcón, profesora actualizada, rigurosa en su trabajo, abierta a lo nuevo. Todas ellas tenían un tremendo compromiso con la educación parvularia, nos movilizaban para su defensa y desarrollo, y nos inculcaban el ser muy buenas estudiantes (PERALTA, 2020, p. 2).

De esta forma, se graduó de Educadora de Párvulos en 1970, con el primer puntaje de su generación. En el primer lustro de su labor como educadora tomó conciencia que la música era muy importante en su acción pedagógica y "me di cuenta que lo que había aprendido en la Escuela no era suficiente para trabajar con niños" (PERALTA, 2020, p. 2); por lo mismo, ingresó a estudiar en la misma Universidad de Chile, Pedagogía en Educación Musical, titulándose en 1979. Luego, en su afán de perfeccionarse y especializarse en curriculum, realizó el Magíster en Educación, Mención Curriculum en la Pontificia Universidad Católica de Chile, graduándose en 1984; su tesis fue dirigida por otro Premio Nacional y especialista en Curriculum, Dr. Abraham Madgenzo (PERALTA 2019a: 1). Sin embargo, este Magíster, según sus propias palabras:

me hizo curriculista, y allí descubrí junto con la Asociación Chilena de Currículo Educacional y mi gran maestra Viola Soto, que el currículum no es neutro y que implica un seleccionar cultura ${ }^{12}$, y yo me sentí débil en mis conocimientos de Antropología Socio-Cultural, y entré a hacer el Magister en Ciencias Sociales, Mención Antropología Socio-Cultural. Se me abrió un mundo que continúa hasta el presente (PERALTA, 2020, p. 2).

Fue así como, en su alma mater obtuvo el grado correspondiente en 1993; dirigió su tesis la Dra. Esther Grebe. Finalmente, en el 2005 obtuvo su grado de Doctora en Educación en la Universidad Academia de Humanismo Cristiano; su tesis fue dirigida por el Dr. Rolando Pinto y se tituló "Modelo de análisis de la calidad en educación parvularia". A su vez, ha realizado varios cursos y pasantías sobre "Enfoque Antropológico de la Mujer", "Planificación Estratégica", "Reggio Emilia Center and Preschools", Modalidades de Educación Infantil" y "Propuesta para la Primera Infancia de Emy Pikler", tanto en Chile como en Italia, España y Hungría (PERALTA, 2019a, pp. 1-2).

\section{c) Rasgos de la personalidad de María Victoria Peralta}

A lo largo de su fructífera existencia, la Premio Nacional fue desarrollando una personalidad con mucho liderazgo; de ello dan cuenta varias de las personas con las cuales le tocó departir su trabajo. El Subsecretario de Educación del primer gobierno de la transición post dictadura militar (1990-1994) -con quien trabajó María Victoria Peralta en el Ministerio de Educación-, Raúl Allard, señala: "Victoria tiene enormes capacidades intelectuales y académicas vinculadas a la educación pero lo fundacional es su sencillez de vida y sensibilidad social que la hace vibrar con los niños a los que atiende y a las educadoras que apoya" (ALLARD, 2020, p. 3). El Dr. Manuel Silva, académico de la Universidad de Chile -quien la conoce desde 1984 como miembro de la Asociación Chilena de Curriculum (ACHCED)-, acota al respecto:

${ }^{12}$ El 4 y 5 de enero de 1989, la UNESCO realizó en Santiago un Seminario, dirigido por la también Premio Nacional en Ciencias de la Educación, Viola Soto Guzmán, titulado "Identidad de América Latina y sus Bases Culturales". Al autor de este artículo -profesor de filosofía- le tocó participar en un Panel, junto al Dr. Humberto Maturana y la entonces Magíster María Victoria Peralta, en que cada uno expuso su punto de vista desde su área de interés, filosofía, neurociencia y curriculum, respectivamente. Quien escribe y Victoria Peralta se conocían desde 1976, pues ambos trabajaban en la Pontificia Universidad Católica de Chile. 
Una persona muy comprometida con las ideas que genera y profesa. Tiene espíritu de iniciativa, proactividad, fraternidad. Tiene capacidad para organizar y promover trabajo colaborativo, en equipo transdisciplinarios. Entusiasta y buena para tocar guitarra y cantar en grupo [...] siempre llevaba el pandero de la música, y todos formábamos un coro en donde compartíamos momentos que siempre recordamos con afecto (SILVA, 2020, p. 2).

Su exalumna y académica de la Universidad de Chile, que la conoce desde 1981, Dra. Mónica Manhey, recalca:

Es una persona muy estudiosa, organizada y perseverante para llegar a sus metas planteadas. Por ejemplo, en el caso de búsqueda de material para sus investigaciones históricas. Hay que destacar que es muy generosa con sus conocimientos y por ello ha publicado y dado tantas conferencias y charlas (la mayoría gratis). Al trabajar junto a Victoria genera en el equipo buenas relaciones, es alegre, amable y confía en los y las demás personas [...] Gran persona y maestra de maestras (MANHEY, 2020, pp. 1 y 3).

Su compañera desde la universidad (1968) y que han trabajado en varias instituciones juntas, Magíster Ester Hernández, subraya sobre la Premio Nacional en Ciencias de la Educación, que es:

Inteligente, despierta, extraordinariamente curiosa, buena para hacer asociaciones, reflexiva, trabajadora incansable, empeñosa hasta alcanzar la meta aunque merme su bienestar o su salud. Líder, correcta, firme. Segura, serena, amable. Buena amiga de las amigas, leal y bondadosa. Da sugerencias en las tribulaciones ajenas si uno se lo pide. Con amor a la naturaleza y a los animales. Con cierto candor juvenil frente al arte. Y con gran sentido del humor en la intimidad (HERNÁNDEZ, 2020, p. 4).

\section{2.- Acción educativa de María Victoria Peralta}

\section{a) Su labor educativa en jardines infantiles y universidades}

Cuando aún no se titulaba, fue creadora, directora y educadora del Jardín Comunitario Peñalolén en la Población Arrieta (1969); educadora en nivel transición en el jardín Infantil Peñalolén (1970); educadora en nivel medio en el Jardín Bambi (1971-1972; educadora en nivel transición en el Jardín Infantil Montahue para trabajadoras de $\operatorname{ENAP}^{13}$ (1973), entre otras instituciones (PERALTA, 2019, pp. 3-4).

En el ámbito académico, se inició como Ayudante (1972) y luego como Profesora (1976) en la Universidad de Chile, ejerciendo hasta 1981, fecha en que fue desvinculada cuando el Instituto Pedagógico se desprendió de la universidad y pasó a ser la Academia de Ciencias Pedagógicas $^{14}$. A su vez, en 1976 ingresó a la Pontificia Universidad Católica de Chile -PUC-, en circunstancias difíciles por la dictadura militar y la presencia de un Rector Delegado en la misma, Almirante Jorge Swett Madge; sobre ello, recuerda:

\footnotetext{
${ }^{13}$ Empresa Nacional de Petróleo, entidad pública.

${ }^{14}$ Medida tomada por la dictadura militar para desmembrar la principal universidad pública del país; le cercenó principalmente las sedes regionales y el Instituto Pedagógico.
} 
Adriana Masses, quien era la coordinadora de la carrera por los años 76, me invitó a ser parte del cuerpo académico e ingresé a dar unas horas, ya que tenía una jornada completa en la Universidad de Chile. Estuve hasta el año 1989, donde pedí permiso por un año para asumir la Vicepresidencia de la JUNJI (1990) y al volver a conversar sobre las posibilidades de hacer clases, me encontré que me había sacado de la nómina. En realidad, todos los años que estuve en la PUC ${ }^{15}$, nunca tuve un contrato por más de 6 meses, por lo que no supe lo que eran vacaciones pagadas [...]. Además, yo me reunía con colegas que no estábamos con la dictadura y participaba en distintas actividades políticas y académicas fuera de la PUC, en CPU ${ }^{16}$, por ejemplo. Esos años no fueron fáciles en la PUC (PERALTA, 2020, p. 3).

Justamente por las razones ya enunciadas, el trabajo académico fue bastante difícil para la Premio Nacional y al cesar en sus funciones en su alma mater, tuvo que ejercer docencia parcializada en diversas instituciones de educación superior en diferentes ciudades del país: Universidad de La Frontera en Temuco (1982); Universidad de La Serena en la misma ciudad (1983-1984); Instituto Profesional Blas Cañas, Instituto Profesional de Providencia e Instituto Profesional del Pacífico (1983-1989), los tres en Santiago y de carácter privado $^{17}$; Universidad de Magallanes en Punta Arenas (1987-1989). Esta situación revela por las peripecias que tuvieron que pasar varios académicos en Chile que no adhirieron a la dictadura militar: se volvieron 'itinerantes' y solo podían ejercer por algunas horas en diferentes instituciones. En sus lugares de trabajo dictó diferentes cátedras: Metodología de la Educación Parvularia, Metodología de la Educación Musical en el Jardín Infantil, Psicopedagogía Infantil y Educación Personalizada (PERALTA, 2019a).

Con la vuelta a la democracia (1990), se reincorporó a su antiguo pedagógico, denominado ahora Universidad Metropolitana de Ciencias de la Educación -UMCE-; allí creó

junto con Mireya Gálvez, el Magister en Educación, mención Educación Parvularia (el primero en Chile), en el posgrado que coordinaba la profesora Viola Soto, en 1994, a los 50 años de haberse creado la carrera universitaria en Chile. Cuando a ella, le pidieron su cargo, que yo consideré que era una injusticia dada su entrega y calidad académica, renuncié a seguir trabajando en la UMCE de aquel tiempo ${ }^{18}$ (PERALTA, 2020, p. 4).

Finalmente, por su contacto con la labor pública por 16 años, se percató de la necesidad de que hubiera más educadoras de párvulos con posgrado para que fueran capaces de investigar e innovar sobre educación infantil. Por ello,

hice un proyecto mejorando algunos aspectos que habíamos evaluado en la UMCE, y lo llevé a la Decana de la Facultad de Educación (de la Universidad Central ${ }^{19}$ ), Selma Simonstein, quien con un grupo de profesores había emigrado de la Universidad de Chile, formando la carrera

\footnotetext{
${ }^{15}$ Pontificia Universidad Católica de Chile.

${ }^{16}$ Corporación de Promoción Universitaria; ONG dedicada al estudio investigativo y difusión de propuestas educativas a nivel universitario preferentemente, pero también del sistema escolar y de la primera infancia.

${ }^{17}$ Las tres universidades en que trabajó por horas durante este período eran públicas.

${ }^{18}$ Ello sucedió en el año 2000.

${ }^{19}$ Universidad privada existente desde 1982 en Chile. Fue la institución que patrocinó su postulación al Premio Nacional en Ciencias de la Educación 2019.
} 
allí, y que era la Presidenta Mundial de $\mathrm{OMEP}^{20}$, además. Era el mejor lugar donde se podría considerar con seriedad la educación parvularia, y con el espíritu de la Universidad de Chile. Se aprobó el proyecto por parte de la Facultad y la Junta Directiva, y se inició el Magister en Educación Infantil, creándose posteriormente el Instituto Internacional de Educación Infantil, que dirijo hasta el presente [...]. Permanezco en esta universidad porque he tenido la libertad para impulsar todo tipo de proyectos interesantes y aportadores para el país (PERALTA, 2020, p. 5).

La Dra. Peralta sigue trabajando académicamente en la Universidad Central. Otro hecho importante en la vida de esta educadora fue su participación activa en la Asociación Chilena de Curriculum -ACHCED-, entidad fundada por importantes académicos durante la dictadura militar en la década del 70 del siglo pasado; algunos de sus Presidentes han sido, entre otros, el Dr. Gilberto Calvo y los Premios Nacionales Abraham Magendzo y Viola Soto Guzmán. Esta última educadora realizaba eventos y reunía a sus asociados en forma permanente. Por lo mismo, el Dr. Manuel Silva recuerda que "[...] la ACHCED, con el liderazgo intelectual y afectivo de la Prof. Viola Soto, nos congregó de manera permanente. Ella fue el nexo que nos aglutinó en la amistad, la actividad académica y la sociabilidad como fuente de encuentro y crecimiento personal e intelectual" (SILVA, 2020, p. 1).

\section{b) Su trabajo por la infancia a nivel privado y público, nacional e internacional}

Durante la dictadura militar, María Victoria, junto a dos socias, Ester Hernández y Gerda Schmid ${ }^{21}$-Educadoras de Párvulos iguales que ella-, fundaron el Centro de Perfeccionamiento, Investigación y Desarrollo Curricular, conocido por la sigla PARVUS ; ello sucedió en los primeros días de agosto de 1981 y el 14 del mismo mes se iniciaron las actividades de perfeccionamiento para educadoras de párvulos, puesto que en 1980 las carreras de pedagogía se eliminaron de las universidades y pasaron a depender de los Institutos Profesionales; la Dra. Peralta

reunió a un grupo de educadoras y nos planteó la necesidad de fundar un centro dónde se pudiesen reunir Educadoras de Párvulos a reflexionar y, además, impartir conocimientos actualizados sobre la educación de los niños pequeños, labor que antes realizaban las universidades" (HERNÁNDEZ, 2020, p. 1).

Esta importante institución se mantuvo hasta 1993. Según una protagonista de la época, "trabajaron incansablemente habilitando el espacio en Avda. Salvador, el que posteriormente se cambió a una casa arrendada en Román Díaz esquina Eleodoro Yáñez" (MANHEY, 2020, p. 3). El funcionamiento de la institución lo describe así una de las socias:

En sesudas reuniones de trabajo [...] siempre dirigidas por Victoria, quien llevó la voz cantante, la de las nuevas ideas y organización de los lugares y los tiempos. Nosotras teníamos estudios de posgrado y por años docentes universitarias. Posteriormente, otras académicas dictaron cursos de su especialidad pagados por PARVUS. El horario de atención era de 18 a 21 horas y se esperaba a las Educadoras-alumnas con un café y galletas porque

\footnotetext{
${ }^{20}$ Es la Organización Mundial para la Educación Preescolar.

${ }^{21} \mathrm{Al}$ cabo de unos años se retira y es reemplazada por la Educadora de Párvulos Jazmín Miranda.
} 
venían de su trabajo. Teníamos una secretaria para tipiar los documentos que apoyaban los nuevos conocimientos, originales de nuestra autoría. Posteriormente, un estudiante de bibliotecología pagado, clasificó y ordenó la biblioteca con nuestros propios libros (HERNÁNDEZ, 2020, pp. 1-2).

Existía una biblioteca y a las alumnas ${ }^{22}$ se les entregaba material preparado por las propias docentes que dictaban los cursos; se cobraba una matrícula para solventar los gastos y los cursos eran semestrales, una vez a la semana y con una duración de 40 horas, certificados para la carrera docente ${ }^{23}$. Había difusión a través de un díptico que

especificaba que PARVUS era un centro educacional con orientación humanista, abierto a todas las ideas y posturas educacionales que consideraban al niño como centro de su acción. Su objetivo era ofrecer un lugar de encuentro, diálogo y reflexión en torno a la problemática de la educación del párvulo, y se especificaban los diversos fines que perseguía (HERNÁNDEZ, 2020, p. 2).

En síntesis, PARVUS era (i) un Centro de perfeccionamiento con cursos en Santiago y regiones ${ }^{24}$, con cursos a distancia con metodología personalizada y que dictaba charlas abiertas a todo público que deseara conocer la educación parvularia; (ii) un Centro de Investigación que realizaba y publicaba pesquisas sobre el área; (iii) un Centro de Desarrollo Curricular que preparaba programas de atención al párvulo con diferentes metodologías y sistemas de gestión y evaluación; efectuaba asesorías metodológicas a instituciones que lo requirieran; elaboraba material didáctico para jardines infantiles y se realizaban publicaciones, entre ellas el Boletín PARVUS $^{25}$ (HERNÁNDEZ, 2020). Por otra parte,

también se organizó un Jardín Infantil modelo para poner en práctica los principios, métodos y postulados de PARVUS ${ }^{26}$ a cargo de la joven educadora Mónica Manhey, destacada discípula en su formación de pregrado de Victoria y Ester. Este Jardín recibía niños de 2 años con un plan de trabajo que los padres conocían de antemano y consecuente con la nueva modalidad de trabajo innovador. La familia recibía informe diario de su hijo e hija [...] era un Jardín de puertas abiertas. La Educadora, cuando el tiempo lo permitía, sacaba a los niños para que conocieran la comunidad y, después,

\footnotetext{
${ }^{22}$ Las alumnas eran educadoras provenientes de Jardines Infantiles, escuelas, centros educativos y otros, que atendían a niños pequeños, estudiantes universitarias de los últimos años de la Carrera o de carreras afines, docentes universitarias jóvenes, todas aportaban algo en relación con el tema que se estaba tratando (HERNÁNDEZ, 2020).

23 Ello significaba que estaban reconocidos por el Centro de Perfeccionamiento, Experimentación e Investigaciones Pedagógicas -CPEIP- del Ministerio de Educación.

${ }^{24}$ Se firmaron Convenios con la Dirección Regional de Educación Provincial de la Primera Región en Arica, la Universidad de la Serena, la Universidad de Talca, la Universidad José Santos Ossa de Antofagasta, la Universidad de Magallanes en Punta Arenas, la Asociación de Educadoras de Párvulos de Calama y con un colegio particular de Rancagua (HERNÁNDEZ, 2020).

${ }^{25}$ Ejercía como Coordinadora de los cursos de perfeccionamiento y de la biblioteca, María Victoria Peralta Espinosa. Como Coordinadora de publicaciones y línea de investigación, Ester Hernández Castillo. Como Coordinadora administrativa, asesorías y material didáctico, Gerda Schmid Spilker, reemplazada posteriormente por Jazmín Miranda. "Todos estos roles fueron asignados por Victoria quién siempre ejerció las relaciones públicas de PARVUS, dedicando mayores horas de trabajo y asumiendo la jefatura del mismo" (HERNÁNDEZ, 2020, p. 3).

${ }^{26}$ En el año 1985 se formó un Jardín Infantil con el fin de ofrecer una Educación Personalizada y ser un centro de Innovación donde se pudieran llevar a cabo prácticas producto de lo investigado por el PARVUS y las profesionales que en él se habían incorporado (MANHEY, 2020).
} 
conversaban de lo visto y realizaban actividades en relación al tema que abarcaba una Unidad completa (HERNÁNDEZ, 2020, p. 4).

María Victoria Peralta llegó al sector público con la vuelta a la democracia en 1990 y asumió como Vicepresidenta Ejecutiva de la JUNJI ${ }^{27}$ (1990-1998) ${ }^{28}$, institución en la cual ““....] se inició la modernización administrativa y técnica [...], se amplió la cobertura [...], se mejoró la calidad de los jardines infantiles y se crearon la mayoría de los programas no-formales [...], entre ellos, los de atención a párvulos de comunidades indígenas" (PERALTA, 2019, p. 7). Su jefe directo en los tres primeros años y medio, el Subsecretario Raúl Allard, señala que "[...] fue una extraordinaria funcionaria. Victoria ya en esos años era la persona que lideraba en el país el nivel parvulario, lo que se ha reafirmado tres décadas después con el Premio Nacional de Educación" (ALLARD, 2020, p. 1).

La dictadura militar devolvió el gobierno a la democracia con una cobertura de la educación de la primera infancia con solo el $28 \%$ de los niños menores de 6 años; pero, a su vez, si se analizaba tal porcentaje, se concluía que los niños entre 2 y 6 años que recibía atención educativa acorde a su edad, el $60 \%$ de ellos era de condición económica media o media alta (Ministerio de Educación, 2001); por lo mismo, el aporte que realizó la Dra. Peralta a la educación parvularia y a los infantes de familias más desfavorecidas, mientras dirigió la Junta Nacional de Jardines Infantiles, fue notable; su labor se proyectó más allá de la institución que dirigía

encarnó a nivel del Gobierno de restauración democrática de Patricio Aylwin la necesidad de reforzar el estatus y la importancia de la educación parvularia en la vida de los niños, la importancia como nivel de la educación, y la importancia de definir una política nacional de calidad y expansión de este nivel que incluía, naturalmente, reforzar la formación y condiciones de trabajo de las educadoras de párvulos. Eso lo pudimos hacer porque estaba Victoria con nosotros en el Gobierno y en el Ministerio (ALLARD, 2020, p. 1).

Específicamente, la Premio Nacional lideró la política del gobierno democrático en lo concerniente a JUNJI e INTEGRA ${ }^{29}$, como asimismo el perfeccionamiento y la ampliación del nivel inicial, en general. En los inicios se identificaron algunas poblaciones metas: párvulos en situación de marginalidad; párvulos en extrema pobreza con riesgo bío-médico y un cuadro social grave; párvulos de madres jefas de hogar que trabajan fuera y en especial, por cuenta propia y con recursos escasos; párvulos de grupos étnicos marginados cultural y socialmente. Por otra parte, se implementaron jardines familiares no convencionales, para atender a niños de dos a cinco años en convenio con municipalidades y organizaciones sociales y comunitarias. Como política pública, la Dra. María Victoria Peralta determinó dos importantes situaciones: (i) el efecto que la educación parvularia tiene en la población de 0 a cinco años, y (ii) el potencial estimulador que este tipo de educación provoca para mitigar desigualdades de los infantes de los estratos socio-económicos más bajos (ALLARD, 2020).

A pesar del esfuerzo realizado, la Educadora Victoria Peralta recuerda que mientras ejerció como Coordinadora Nacional de Educación Preescolar del Ministerio de Educación

\footnotetext{
${ }^{27}$ La Junta Nacional de Jardines Infantiles -JUNJI- fundada en 1970 a través de la Ley $\mathrm{N}^{\circ} 17.301$ durante el gobierno de Eduardo Frei Montalva (CAICEO, 2011).

${ }^{28}$ Nombró Directora Nacional del Departamento Técnico Nacional a Ester Hernández Castillo.

${ }^{29}$ Fundación creada en 1990, como una institución de derecho privado sin fines de lucro, en apoyo a los sectores de menores recursos y, especialmente para los hijos de la mujer trabajadora, que pertenece a la Red de Fundaciones de la Presidencia de la República; fue una de las primeras medidas de política pública con la vuelta de la democracia (CAICEO, 2011).
} 
(1998-2001), tuvo que enfrentar a funcionarios estatales que pensaban que era innecesario preocuparse de la enseñanza de los pequeños; textualmente, señala:

Me tocó escuchar comentarios del tipo 'pero ¡cómo van a hacer un currículum para las guaguas, si ese es un tema de pañales y mamaderas!'. Dolían esas cosas, porque no se reconocía el derecho a la educación del niño. Pero ahora es distinto, hemos visto un mayor apoyo y consideración a nuestra área (PERALTA, 2019b: s/p).

Al dejar la Vicepresidencia de la JUNJI, pasó a coordinar el equipo que establecerá "las Bases Curriculares de la Educación Parvularia"30, manteniéndose como Asesora de la JUNJI (1998-2001) y, posteriormente, como Coordinadora de la Unidad de Educación Parvularia en el Ministerio de Educación (2002-2006). De esta forma, estuvo 16 años en el servicio público.

Por otra parte, a nivel internacional, la Dra. Peralta ha realizado labores de consulta para diversas organizaciones internacionales, como la OES ${ }^{31}$, el UNICEF, la UNESCO, el BID, el PMA y la OEI, en el ámbito de la educación infantil. Además ha sido miembro del Comité de Expertos de los Socios Latinoamericanos de la Organización de Estados Iberoamericanos -OEIy, posteriormente, fue elegida Vicepresidenta de la Organización Mundial para la Educación Preescolar -OMEP ${ }^{32}$ - para América Latina durante el período 2008-2010 (UNESCO, s/f). A su vez, en otros países latinoamericanos ha sido asesora de diversos tipos de proyectos de: Planes nacionales para la primera infancia, mejoramiento de la calidad, de construcción de currículos nacionales en educación inicial y formadora de especialistas (Paraguay, Ecuador, República Dominicana, Panamá, Uruguay) (PERALTA, 2019).

\section{c) Rol como educadora de la Dra. Peralta}

María Victoria Peralta es especialista en materias de Curriculum y Cultura e Historia de la Educación. Para el Dr. Manuel Silva (2020, p. 2), su labor

constituye un aporte sustantivo a la educación chilena y latinoamericana. Sus acciones pedagógicas tienen el sello de la búsqueda de la pertinencia cultural como impronta de la acción educativa intencionada. Ha tenido participación significativa en las Reformas a la Educación Parvularia en Chile, y la mayoría de los países latinoamericanos, donde han reconocido su valioso aporte [...]. También en lo laboral, su aporte a la construcción de Curricula culturalmente pertinentes, generando y acompañando equipos de educación parvularia, en diversas comunas del país. Ello es una muestra de construcción de Curriculum, desarrollando las capacidades presentes en las educadoras para el diseño de planes, programas, materiales y actividades que aglutinan lo local con lo regional y lo nacional.

Para su exalumna, la Dra. Mónica Manhey (2020, p. 4), su maestra

es una educadora $100 \%$, no sólo de aula o al trabajar remuneradamente, en su vida real y cotidiana, en la calle, en su relación con las personas día a día. Eso lo he visto en los años que nos conocemos. De una enorme

\footnotetext{
${ }^{30}$ Las cuales se promulgaron en el 2001; en el 2018 se han actualizado por los cambios teóricos existentes en estos años y por la nueva realidad imperante.

${ }^{31}$ Organización para la Excelencia de la Salud.

${ }^{32}$ En Chile se había creado el Comité respectivo en 1948.
} 
sensibilidad ante los niños y niñas. Cuando nos hacía docencia enseñaba teoría y práctica, cargaba con cajas de instrumentos para la clase de música y nos enseñó mucho que no conocíamos. Todas sus clases siempre fueron imperdibles, ya en esos años se acompañaba de medios audiovisuales, nos mostraba fotos de modalidades curriculares, de niños y niñas aprendiendo, de prácticas innovadoras... ello en cada una de las clases de pregrado que tuve con ellas, así también en mis estudios de Magíster que también fui su estudiante. Nos 'paseaba' por diferentes partes de Chile y otros países, siempre con un sentido formativo.

Para su compañera de estudios, socia y amiga, Ester Hernández (2020: 5), María Victoria

fue y aún es una educadora, en todo el sentido de la palabra, extraordinaria. Innovadora en la presentación del tema con recursos visuales que ella seleccionaba o elaboraba \{fotografías, música, artesanías, libros, documentos $\}$ que traía de sus viajes a países ciudades pueblos y villorrios a raíz de los cursos y asesorías que impartía en Instituciones públicas y privadas y universidades que requerían sus servicios. Además, presentaba investigaciones propias y sugería nuevas, en seminarios, simposios y otros encuentros. No obstante donde permanentemente fue requerida su presencia fue para dar cursos certificados para profesionales y estudiantes universitarios deseosos de escuchar los nuevos enfoques en educación y recibir su carpeta con documentos y nuevos planteamientos curriculares que ya se estaban implementando en Chile, poniendo al niño en el centro del proceso y no al educador.

\section{Conclusiones}

Como ha podido apreciarse, la Educadora de Párvulos, María Victoria Peralta ha sido y sigue siendo una maestra a cabalidad que se fue transformando en la líder de la educación parvularia en Chile y Latinoamérica. Con diversos premios de reconocimiento en varios países latinoamericanos e incluso europeos, coronando su importante labor con el Premio Nacional de Ciencias de la Educación en su país.

Hablar de Educación Parvularia en Chile y en Latinoamérica conduce necesariamente a la figura y vastos escritos de esta insigne educadora con pensamiento propio, el cual será analizado en otra ocasión.

Recientemente ha luchado por la sala cuna universal y gratuita para toda mujer trabajadora y padre que esté a cargo de un hijo menor; al respecto, ha diferido del actual proyecto que el Presidente Piñera (2018-2021) ha presentado en Chile, igual que la Comisión de Educación del Senado, porque "no existe universalidad real, se mantiene un sesgo en la perspectiva de género, se vuelve al lucro en la educación temprana" (SENADO DE LA REPÚBLICA DE CHILE, 2019: s/p).

\section{Referencias}

AA. (2019). María Victoria Peralta, primera educadora de párvulos galardonada con el Premio Nacional de Educación en https://www.mineduc.cl/2019/08/27/maria-victoria-peralta-premionacional-de-educacion-2019/. Consultado el 20 de diciembre de 2019.

ALLARD, Raúl (2020). Entrevista. Académico de la Pontificia Universidad Católica de Valparaíso y Subsecretario de Educación, realizada por el autor de este artículo en Valparaíso, Chile, 6 de enero. 
ARANCIBIA, Elena; CAICEO, Jaime; RETAMAL, Myriam (2004). Historia de los 150 años de las Hijas de la Caridad y de la Congregación de la Misión en Chile. Santiago: Colegio Santa Familia.

CAICEO, Jaime (2011). Desarrollo de la Educación Parvularia en Chile. Revista História da Educação, V. 15(34), 22-44. No tiene DOI; se le ubica en https://seer.ufrgs.br/asphe/article/view/21273/12249. Consultado el 15 de diciembre de 2019.

CAICEO, Jaime (2015). Amanda Labarca, Importante Educadora Feminista del Siglo XX en Chile. Cadernos de História da Educação, Vol. 14(3), pp. 915-930. No tiene DOI; se le ubica en http://www.seer.ufu.br/index.php/che/article/ view/33144/17847. Consultado el 20 de diciembre de 2019.

CAICEO, Jaime (2018). El Porqué del Desarrollo Insuficiente de la Filosofía de las Ciencias Sociales. El Futuro del Pasado, n.9, pp.393-418. https://doi.org/10.14516/fdp.2018.009.001.014

CORNEJO, Marcela (2006). El Enfoque Biográfico: Trayectorias, Desarrollos Teóricos y Perspectivas. PSYKHE, v.15(1), pp.95-106. https://doi.org/10.4067/S0718-22282006000100008

HERNÁNDEZ, Ester (2020). Entrevista. Académica de la Universidad de Chile y colega en Parvus, realizada por el autor de este artículo en Santiago de Chile, 14 de enero.

MANHEY, Mónica (2020). Entrevista. Académica de la Universidad de Chile y exalumna, realizada por el autor de este artículo en Santiago de Chile, 13 de enero.

MINISTERIO DE EDUCACIÓN (2001). La Educación Parvularia en Chile. Santiago de Chile: División de Educación General/Unidad de Educación Parvularia.

PERALTA, María Victoria (2006). Cien años de Educación Parvularia en el sistema Público. El primer Kindergarten Fiscal. 1906-2006. Santiago: Universidad Central.

PERALTA, María Victoria (2019a). Curriculum Vitae. Entregado al Ministerio de Educación para postular al Premio Nacional de Educación. Santiago de Chile: MINEDUC.

PERALTA, María Victoria (2019b). Las educadoras queremos que reconozcan la importancia de nuestro trabajo en https://www.explora.cl/blog/las-educadoras-queremos-que-reconozcanla-importancia-de-nuestro-trabajo/. Consultado el 7 de diciembre de 2019.

PERALTA, María Victoria (2020). Entrevista. Protagonista del texto, realizada por el autor de este artículo en Santiago de Chile, 7 de enero.

SENADO DE LA REPÚBLICA DE CHILE (2019). Comisión de Educación rechaza Sala Cuna Universal en https://www.senado.cl/comision-de-educacion-rechaza-sala-cunauniversal/senado/2019-11-06/172347.html. Consultado el 15 de diciembre de 2019.

SILVA, Manuel (2020). Entrevista. Académico de la Universidad de Chile y colega en la Sociedad Chilena de Curriculum, realizada por el autor de este artículo en Santiago de Chile, 6 de enero.

SUBSECRETARÍA DE EDUCACIÓN PARVULARIA (2018). Bases Curriculares Educación Parvularia. Santiago de Chile: Ministerio de Educación.

UNESCO (s/f). María Victoria Peralta, Chile en http://www.unesco.org/new/es/ world-conferenceon-ecce/expert-commitee/maria-victoria-peralta-chile/. Consultada el 26 de enero de 2020. 